\title{
Searching for high speed long-lived charged massive particles at the LHC
}

\author{
Jie Chen ${ }^{\mathrm{a}}$, Todd Adams ${ }^{\mathrm{b}}$ \\ Department of Physics, Florida State University, Tallahassee, FL 32306, USA
}

Received: 27 January 2010 / Revised: 2 March 2010 / Published online: 24 March 2010

(C) Springer-Verlag / Società Italiana di Fisica 2010

\begin{abstract}
The conventional way to search for long-lived CHArged Massive Particles (CHAMPs) is to identify slow (small $\beta$ ) tracks using delayed time of flight and high ionization energy loss. But at the 7-14 $\mathrm{TeV}$ center of mass energy of the LHC, a CHAMP may be highly boosted (high $\beta$ ) and therefore look more like a minimum ionizing particle, while for high momentum muons $(\gtrsim 500 \mathrm{GeV} / \mathrm{c})$ the radiative effect dominates energy deposition. This suggests a new strategy to search for CHAMPs at the LHC. Using energy deposition from different detector components, we construct a boosted decision tree discriminant to separate high momentum CHAMPs from high momentum muons. This method increases substantially the CHAMP discovery potential and it can be used to distinguish possible di-CHAMP or CHAMP-muon resonance models from dimuon resonance models. We illustrate the new method using a mGMSB model and a recently proposed di-CHAMP model and we give updated CHAMP mass limits for these two models using the results from a recent CDF CHAMP search.
\end{abstract}

\section{Introduction}

Many extensions of the standard model (SM) suggest the existence of a CHArged Massive Particle (CHAMP) that is long-lived [1-8]. The long lifetime is caused by limited phase space due to small mass splittings or by small couplings. If the CHAMP escapes a particle detector before decaying it can be regarded as stable. CHAMP searches play an important role in constraining SUSY models (see, for example, [9]). Therefore, there is interest in pursuing these searches using early LHC data.

A number of searches for weakly-interacting CHAMPs were performed at LEPII [10-13], yielding 95\% C.L. lower

\footnotetext{
a e-mail: jchen@fnal.gov

be-mail: tadams@hep.fsu.edu
}

mass limits of $\sim 100 \mathrm{GeV} / \mathrm{c}^{2}$ for a stable supersymmetric partner of SM leptons [14]. Tevatron searches for CHAMPs have been carried out recently by CDF [15] and D0 [16]. The conventional method to detect CHAMPs is to search for slow moving and/or highly ionizing particles [17]. This technique has been used for the LEP and Tevatron searches, where the CHAMPs are expected to be slow due to the low center of mass energy and the expected high CHAMP mass $\left(>100 \mathrm{GeV} / \mathrm{c}^{2}\right)$.

At the energy of the LHC $(\sqrt{s}=7-14 \mathrm{TeV})$, however, CHAMPs can be highly boosted. Therefore, they behave more like minimum ionizing particles (MIPs), and consequently, the conventional search technique may lead to significant signal losses. Current experimental efforts at the LHC $[18,19]$ focus on slow moving CHAMPs, separating them from muons using their larger ionization energy loss and time of flight delays. In this paper, we propose a way to search for high speed CHAMPs. We use the difference in energy deposition in the sub-detectors, as high speed (high momentum, $p$ ) CHAMPs behave like MIPs while high $p$ muons tend to deposit more energy due to radiation effects. The goal is to increase the discovery potential of the LHC experiments using early data.

\section{Models under investigation}

We investigate two models for this study: the minimal gauge mediated supersymmetry breaking (mGMSB) model [1] and a resonant di-CHAMP model [6]. In all simulations, we use $\sqrt{s}=10 \mathrm{TeV}$ for the LHC and $\sqrt{s}=2 \mathrm{TeV}$ for the Tevatron. The mGMSB model we explore corresponds to the SPS7 benchmark scenario proposed in [20]. In this model, the stau $(\tilde{\tau})$ is the next-to-lightest supersymmetric particle (NLSP). We vary $\Lambda$ from $31 \mathrm{TeV}$ to $100 \mathrm{TeV}$, with fixed parameters $N_{\text {mes }}=3, \tan \beta=15, \mu>0, C_{\text {grav }}=10000$ and $M_{\text {mes }} / \Lambda=2$. The large value of $C_{\text {grav }}$ results in a longlived stau, while $\Lambda=31-100 \mathrm{TeV}$ gives a stau mass of 

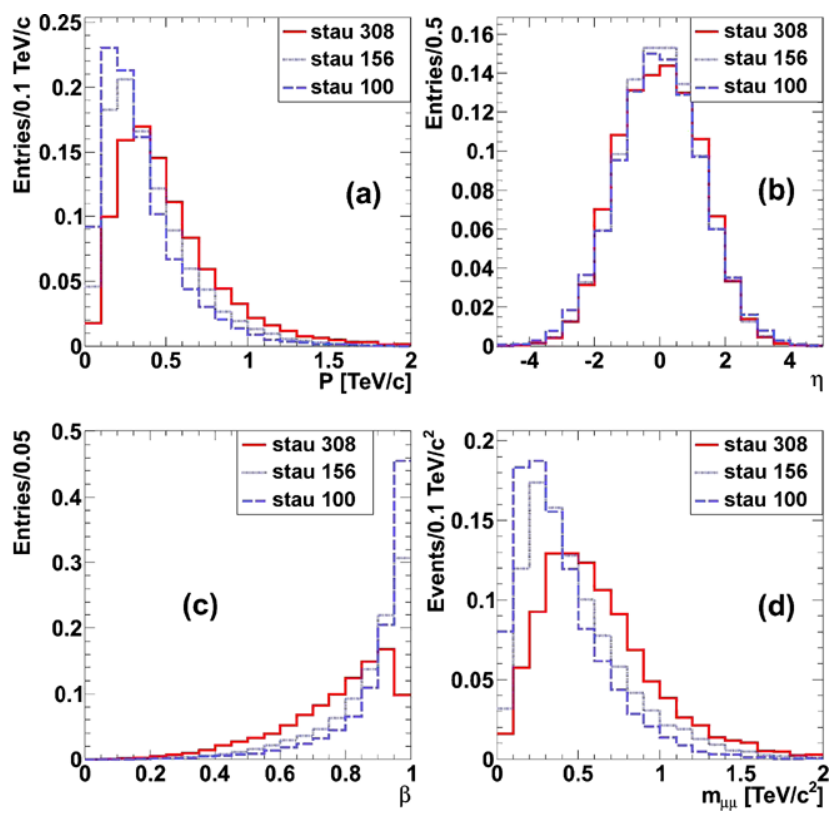

Fig. 1 Distributions for some kinematic quantities for the SPS7 benchmark scenario at stau masses of 100,156 , and $308 \mathrm{GeV} / \mathrm{c}^{2}$ for: (a) stau $p$, (b) stau $\eta$, (c) stau $\beta$, (d) di-stau invariant mass (assuming track has zero mass). All distributions are normalized to unit area

$100 \mathrm{GeV} / \mathrm{c}^{2}$ to $308 \mathrm{GeV} / \mathrm{c}^{2}$. We produce the mGMSB particle mass spectrum and the decay table with the program ISASUGRA [21], which is used as input to PYTHIA 6.4 [22] with all SUSY processes enabled. Figure 1 shows the distribution for some kinematic quantities for three different values of the stau mass within this benchmark scenario, at an LHC energy of $10 \mathrm{TeV}$.

Some recent models predict di-CHAMP [6], or CHAMPmuon [7] resonances in which the CHAMPs are relatively light compared to the resonances, and thus obtain a large boost. We generate di-CHAMP events using COMPHEP 4.5.1 [23] with a model file provided by Kilic, Okui and Sundrum based on [6]. The COMPHEP events are hadronized using the program PYTHIA. We vary the $M_{\tilde{\rho}}$ parameter in the di-CHAMP model from $1.0 \mathrm{TeV} / \mathrm{c}^{2}$ to $2.5 \mathrm{TeV} / \mathrm{c}^{2}$, yielding CHAMP $(\tilde{K})$ masses between $121 \mathrm{GeV} / \mathrm{c}^{2}$ and $302 \mathrm{GeV} / \mathrm{c}^{2}$. The $\tilde{K}$ to $\tilde{\rho}$ mass ratio is 0.12 in the model used and the factorization scale is set to the invariant mass of the di-CHAMP system. Figure 2 shows the distribution for some kinematic quantities for this model at $10 \mathrm{TeV}$.

Both models predict events that may contain two CHAMPs. The di-CHAMP invariant mass distributions are shown in Figs. 1 and 2. Because high $\beta$ CHAMPs resemble muons in their experimental signatures (for example, they are deeply penetrating and have a short travel time to the detectors), these events could appear in searches for high mass di- $\mu$ resonances (as predicted by $Z^{\prime}$ models [24-27]) or in the high mass tails of di- $\mu$ distributions (as in contact interaction models $[28,29])$.
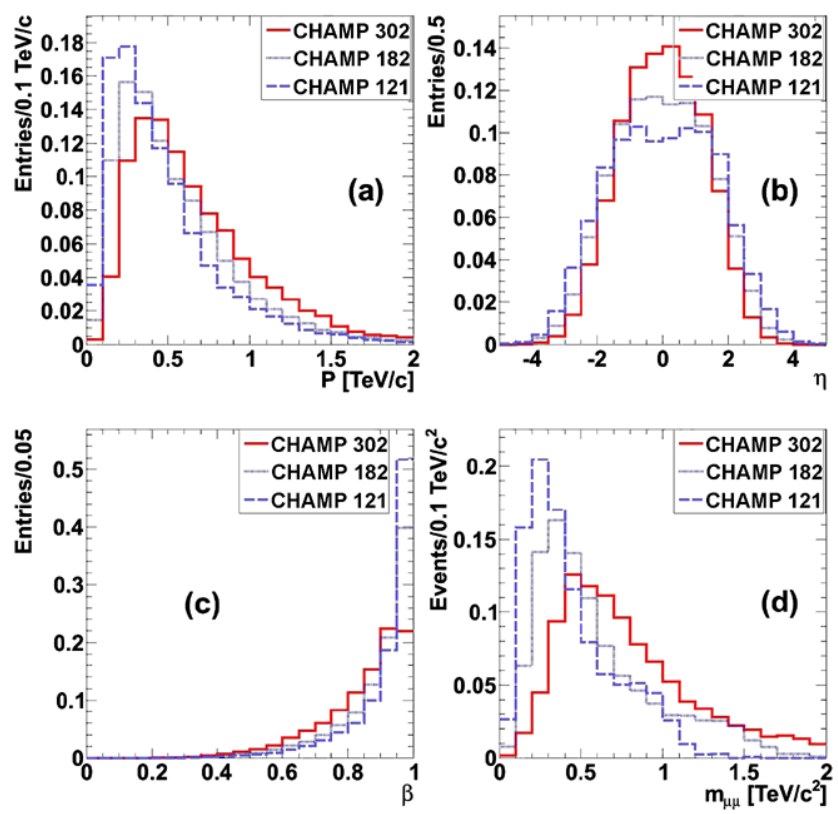

Fig. 2 Distributions for some kinematic quantities for the di-CHAMP model with CHAMP mass 121,182 and $302 \mathrm{GeV} / \mathrm{c}^{2}$ for: (a) CHAMP $p$, (b) CHAMP $\eta$, (c) CHAMP $\beta$, (d) di-CHAMP invariant mass (assuming track has zero mass). All distributions are normalized to unit area

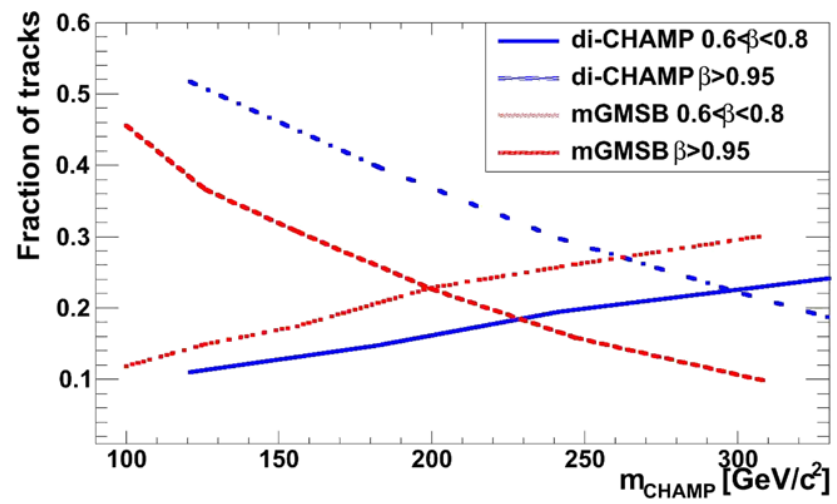

Fig. 3 (Color online) The fraction of tracks that fall within $\beta>0.95$ and $0.6<\beta<0.8$ for the mGMSB stau model (red) and the di-CHAMP model (blue) at $10 \mathrm{TeV}$ LHC energy

We define $\beta>0.95$ as the high momentum (muon-like) region and $0.6<\beta<0.8$ as the traditional CHAMP region. The $0.6-0.8 \beta$ range is the approximate range of the planned LHC experimental searches. Both ATLAS and CMS have acceptance for $\beta<0.6$, but the efficiency falls rapidly $[18,19]$ below this value. Figure 3 shows the fraction of events in the two regions for the mGMSB and di-CHAMP models at $10 \mathrm{TeV}$. For both models, more events are produced in the high momentum region than in the traditional search region for CHAMPs of lower mass. Given the expected higher trigger and reconstruction efficiency for the high momentum region than for the traditional CHAMP search region, we would expect to observe more high mo- 


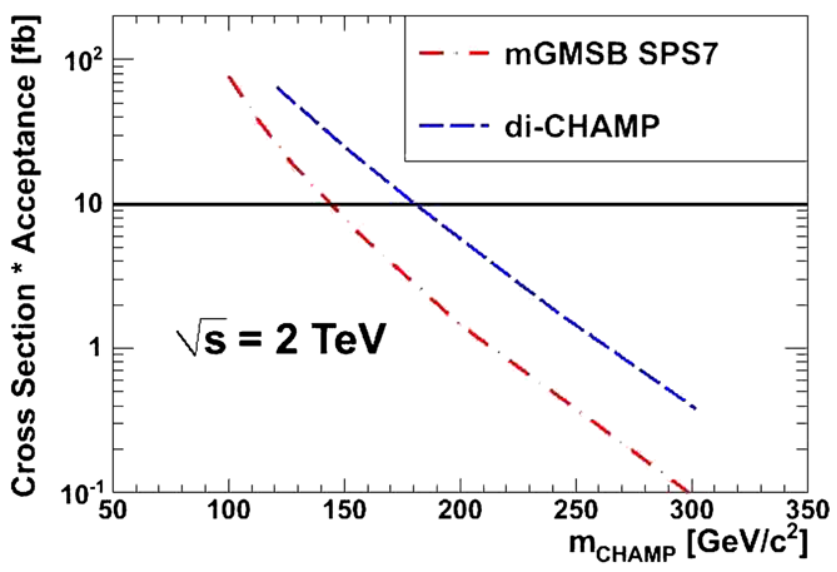

Fig. 4 (Color online) Cross section times acceptance for the mGMSB SPS7 benchmark scenario (red) and di-CHAMP model (blue) at $\sqrt{s}=2 \mathrm{TeV}$. The horizontal black line shows the CDF limit on single, weakly-interacting CHAMPs [15]

mentum than low momentum CHAMPs when their masses are low.

Previous experimental results from LEP and the Tevatron limit the interesting phase space for high momentum CHAMPs at the LHC. CDF has set a limit of $10 \mathrm{fb}$ on the cross section to produce a single, weakly-interacting CHAMP. To apply this limit to our benchmark models, we must account for the fact that some events may have two CHAMP candidates. Therefore, we calculate the acceptance, $\alpha$, for an event to pass the CDF slowly moving minimum ionizing particles trigger criteria $\left(p_{T}>40 \mathrm{GeV} / \mathrm{c}\right.$, $|\eta|<0.7,0.4<\beta<0.9$, and isolation) given one or two chances:

$\alpha=\frac{N_{1}+N_{2} \times \frac{\epsilon_{2}}{\epsilon_{1}}}{N_{\text {tot }}}$,

where $N_{\text {tot }}$ is the total number of events generated, $N_{1}\left(N_{2}\right)$ is the number of events with one (two) CHAMPs within the trigger criteria, $\epsilon_{1}$ is the efficiency given by [15] to find a CHAMP given one chance, and $\epsilon_{2}$ is the efficiency to find a CHAMP given two chances [30].

Figure 4 shows the cross section times acceptance for these models as a function of the CHAMP mass. We interpret this plot as excluding stau masses below $\sim 140 \mathrm{GeV} / \mathrm{c}^{2}$ and di-CHAMP resonances with CHAMP masses below $\sim 180 \mathrm{GeV} / \mathrm{c}^{2}$. Tables 1 and 2 list the cross sections for the two models, and the corresponding CDF acceptance at $\sqrt{s}=2 \mathrm{TeV}$. Based on these exclusion limits, we select the stau mass $=156 \mathrm{GeV} / \mathrm{c}^{2}$ in the mGMSB model and the $\tilde{K}$ mass $=182 \mathrm{GeV} / \mathrm{c}^{2}$ in the di-CHAMP model as our benchmark signals (labeled stau156 and di-CHAMP182 in the following).
Table 1 Cascade and pair production cross sections at Tevatron and LHC energies for the SPS7 benchmark scenario in mGMSB. The last column shows the acceptance for an event to have at least one CHAMP within the CDF analysis selection for cascade production at the Tevatron energy

\begin{tabular}{|c|c|c|c|c|c|}
\hline \multirow{2}{*}{$\begin{array}{l}\text { Mass } \\
\left(\mathrm{GeV} / \mathrm{c}^{2}\right)\end{array}$} & \multicolumn{2}{|c|}{$\sigma(\sqrt{s}=2 \mathrm{TeV})$} & \multicolumn{2}{|c|}{$\sigma(\sqrt{s}=10 \mathrm{TeV})$} & \multirow{2}{*}{$\begin{array}{l}\text { Acceptance } \\
\text { for CDF } \\
\text { analysis }\end{array}$} \\
\hline & $\begin{array}{l}\text { Cascade } \\
\text { (fb) }\end{array}$ & $\begin{array}{l}\text { Pair } \\
(\mathrm{fb})\end{array}$ & $\begin{array}{l}\text { Cascade } \\
\text { (fb) }\end{array}$ & $\begin{array}{l}\text { Pair } \\
\text { (fb) }\end{array}$ & \\
\hline 100 & 113 & 10.6 & 4716 & 57 & 0.68 \\
\hline 112 & 58 & 7.5 & 2433 & 34 & 0.69 \\
\hline 126 & 28 & 4.8 & 1212 & 21 & 0.74 \\
\hline 156 & 7.7 & 1.7 & 320 & 11 & 0.82 \\
\hline 200 & 1.6 & 0.5 & 88 & 4 & 0.90 \\
\hline 247 & 0.43 & 0.2 & 29 & 2 & 0.96 \\
\hline 308 & 0.08 & 0.03 & 9 & 0.8 & 0.96 \\
\hline
\end{tabular}

Table 2 Production cross sections at Tevatron and LHC energies for the di-CHAMP model. The last column shows the acceptance for an event to have at least one CHAMP within the CDF analysis selection at $2 \mathrm{TeV}$ Tevatron energy

\begin{tabular}{llll}
\hline $\begin{array}{l}\text { Mass } \\
\left(\mathrm{GeV} / \mathrm{c}^{2}\right)\end{array}$ & $\begin{array}{l}\sigma(\sqrt{s}=2 \mathrm{TeV}) \\
(\mathrm{fb})\end{array}$ & $\begin{array}{l}\sigma(\sqrt{s}=10 \mathrm{TeV}) \\
(\mathrm{fb})\end{array}$ & $\begin{array}{l}\text { Acceptance } \\
\mathrm{CDF}\end{array}$ \\
\hline 121 & 90 & 845 & 0.71 \\
151 & 30 & 360 & 0.80 \\
182 & 11 & 175 & 0.87 \\
242 & 2.0 & 53 & 0.93 \\
302 & 0.4 & 20 & 0.96 \\
\hline
\end{tabular}

\section{Event generation and selection}

In addition to the signal (see Sect. 2), we model multiple sources of SM (muon) backgrounds ( $W, Z / \gamma^{*}$, multijet, $t \bar{t}$, and diboson) using PYTHIA 6.4. All samples are generated using the CTEQ 5L parton distribution functions (PDFs) [31]. We will use CMS as our reference LHC detector for this study. However, the principles (modified appropriately) are also applicable to ATLAS.

To select CHAMP candidate events, we require at least one high momentum $(p>500 \mathrm{GeV} / \mathrm{c})$ muon-like track within the $|\eta|<1.479$ region. The $500 \mathrm{GeV} / \mathrm{c}$ momentum requirement corresponds to $\beta>0.95$ for the stau156 and $\beta>0.94$ for di-CHAMP182, which are fast enough to appear similar to muons (for masses at the upper edge of the range considered in these models, this requirement corresponds to $\beta \gtrsim 0.85$ ). Moreover, this ensures that background muons are highly boosted and their energy loss is dominated by radiation. The muon critical energy occurs at a muon momentum of $317 \mathrm{GeV} / \mathrm{c}$ in copper, $170 \mathrm{GeV} / \mathrm{c}$ in lead tungstate and $581 \mathrm{GeV} / \mathrm{c}$ in silicon [32]. The $|\eta|<$ 1.479 selection corresponds to the barrel part of the CMS 
Table 3 Cross sections for signal and background samples before selection and after leading and second leading candidate selections. The $W / Z+$ (jets) and multijet backgrounds have generator level cuts of $30 \mathrm{GeV} / \mathrm{c}$ and $215 \mathrm{GeV} / \mathrm{c}$ on the leading jet $p_{T}$, respectively

\begin{tabular}{lccc}
\hline Process & $\sigma(\mathrm{pb})$ & $\begin{array}{l}\geq 1 \text { high } p \mu \\
\sigma(\mathrm{pb})\end{array}$ & $\begin{array}{l}\text { 2nd } \mu \\
\sigma(\mathrm{pb})\end{array}$ \\
\hline$W+$ (jets) & 75600 & 0.3 & 0.002 \\
$Z / \gamma^{*}+($ jets $)$ & 7240 & 0.09 & 0.03 \\
$t \bar{t}$ & 234 & 0.03 & 0.003 \\
$W Z, W W, Z Z$ & 69.4 & 0.005 & 0.001 \\
Multijet & 20100 & $<0.01$ & $<0.01$ \\
Total Background & 103000 & 0.43 & 0.04 \\
stau156 & 0.32 & 0.09 & 0.08 \\
di-CHAMP182 & 0.18 & 0.05 & 0.05 \\
\hline
\end{tabular}

electromagnetic calorimeter (Ecal) [33], where the energy measurement is most accurate. The $\eta$ requirement also helps reject high momentum muon background in the forward region. In addition, we require a second muon-like track with momentum $p>100 \mathrm{GeV} / \mathrm{c}$ and $p_{T}>20 \mathrm{GeV} / \mathrm{c}$ in the $|\eta|<2.5$ region to reduce the $W+$ (jets) background. Table 3 shows the cross sections for the signal and background processes after the leading and second leading $\mathrm{CHAMP} /$ muon requirements.

After these simple selection criteria, the background level is already much smaller than, or similar to, the expected signal for the stau156 and di-CHAMP182 benchmark models. Therefore, even a simple counting experiment may be sensitive to a CHAMP signal. However, due to PDF uncertainties and the fact that PYTHIA is only a tree level generator, the background assumed here may be underestimated. The effect of a larger background will be discussed later. In any case, the energy deposition in the detector can be used to separate further signal from background.

\section{Detector simulation}

We use GEANT4 [34] to simulate a simplified model of the CMS detector [33], shown in Fig. 5. In our model, the tracker is made of 20 layers of $300 \mu \mathrm{m}$ silicon, the electromagnetic calorimeter is a 5 by 5 array of $\mathrm{PbWO}_{4}$ crystal with the same dimensions as a CMS Ecal crystal, and the hadron calorimeter (Hcal) is composed by 30 layers of brass with transverse size similar to that of a CMS Hcal tower. No magnetic field is included in our simulation, as we expect high momentum tracks to be rather straight in the detector.

The energy loss per path length $(d E / d x)$ for a muon or a CHAMP in the tracker is calculated using the truncated mean [35] estimator in which the arithmetic mean is computed after truncation of the highest $40 \%$ of the charge samples for each track. We take the Ecal energy to be the energy

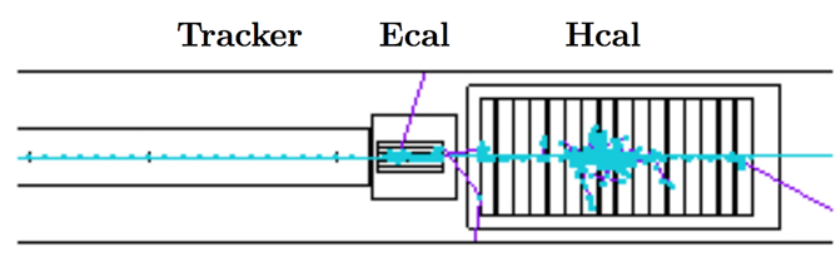

Fig. 5 GEANT4 simulation: a muon track with $p=650 \mathrm{GeV} / \mathrm{c}$ passing through a simplified model of the CMS detector

deposited in the crystal traversed by the CHAMP or muon. This was chosen to minimize the possible energy contribution from nearby charged or neutral particles and to avoid the need to simulate the shower shape in a magnetic field. The Hcal energy is computed by summing the energy measurements for all layers of Hcal, which is roughly the same as the single tower energy defined in the CMS detector.

To better account for the detector response, the GEANT4 simulated energy is smeared with the CMS detector energy resolution functions [33]. For Hcal it is $\sigma / E=120 \% / \sqrt{E}+$ $6.9 \%$ [36]. The Ecal energy is smeared using $(\sigma / E)^{2}=$ $(2.8 \% / \sqrt{E})^{2}+(0.12 / E)^{2}+(0.30 \%)^{2}$. For tracker $d E / d x$, we do not smear the energy because the simulated energy resolution is approximately the same as that of the reconstructed energy. This is due to the very high signal to noise ratio for the CMS tracker readout electronics [33].

As noted in Sect. 3, the main background is $Z / \gamma^{*}+$ (jets) after our simple selections. We save all high momentum tracks from all background or signal events and create background samples by mixing events according to their cross sections. The muon/CHAMP tracks are then passed to our simplified CMS GEANT4 simulation and we obtain the energy deposition for each track.

The results of our simulation and energy smearing indicate that the distributions of CHAMP energies in Ecal and Hcal should be Gaussian (see Fig. 6). This is due to the fact that CHAMPs primarily lose energy through ionization. For the thick material in Ecal and Hcal, the Landau distribution becomes Gaussian. The high speed muons, however, exhibit energy deposition, corresponding to radiative energy losses. For the tracker, the $d E / d x$ distribution is Gaussian in both cases, as expected for the truncated mean estimator [35].

In Fig. 7, we show the average values of Tracker $d E / d x$, energy deposition in Ecal, energy deposition in Hcal, and energy deposition difference between Hcal and Ecal as a function of momentum, for two CHAMP models and two mass points. The low momentum region $(\lesssim 500 \mathrm{GeV} / \mathrm{c}$, corresponding to low $\beta \mathrm{s}$, which is the focus of conventional CHAMP searches) shows the high ionization energy loss, which is inversely proportional to $\beta^{2}$ [32]. We make an important observation: the average energy deposition in Ecal and Hcal in the high momentum region $(\gtrsim 500 \mathrm{GeV} / \mathrm{c})$ are almost identical for the four model/mass benchmarks considered. Note that the muon energy deposition increases 

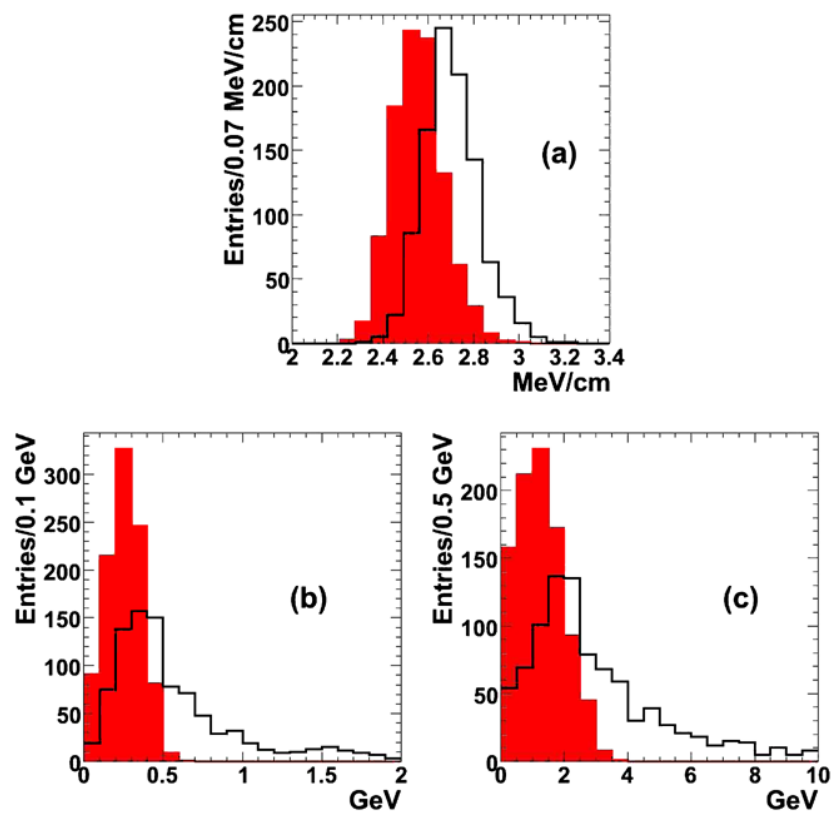

Fig. 6 Distributions of (a) $d E / d x$ in the Tracker, (b) energy deposition in Ecal, and (c) energy deposition in Hcal. The high momentum stau156 signal and the background are shown with a solid and an empty histogram respectively

with momentum, as expected from the increased radiative contributions, while the Tracker $d E / d x$ of muons is evenly distributed in approximately the full momentum range, mainly due to the fact that here $d E / d x$ is calculated with $40 \%$ truncation, which does not take into account high energy (radiative) hits.

Given the important observation that the energy depositions in these detectors is largely model and mass independent in the high $p$ region (Fig. 7), we can use the energies to develop a new method to separate CHAMPs from muons in a manner that is analogous to standard particle identification that separates electrons and photons from hadrons and jets [37]. In the following section we use a multivariate method to enhance the discrimination between muons and CHAMPs.

\section{Multivariate analysis}

The direct use of the energy depositions, whose distributions are shown in Fig. 6, can only provide limited separation between CHAMPs and muons passing our basic selection. However, it is well established that better separation between distributions can often be obtained by combining well understood variables into a multivariate discriminant. To that end, we use the boosted decision tree (BDT) method provided in the Toolkit for Multivariate Data Analysis (TMVA) [38] to compute discriminants that take the three energy depositions as inputs.
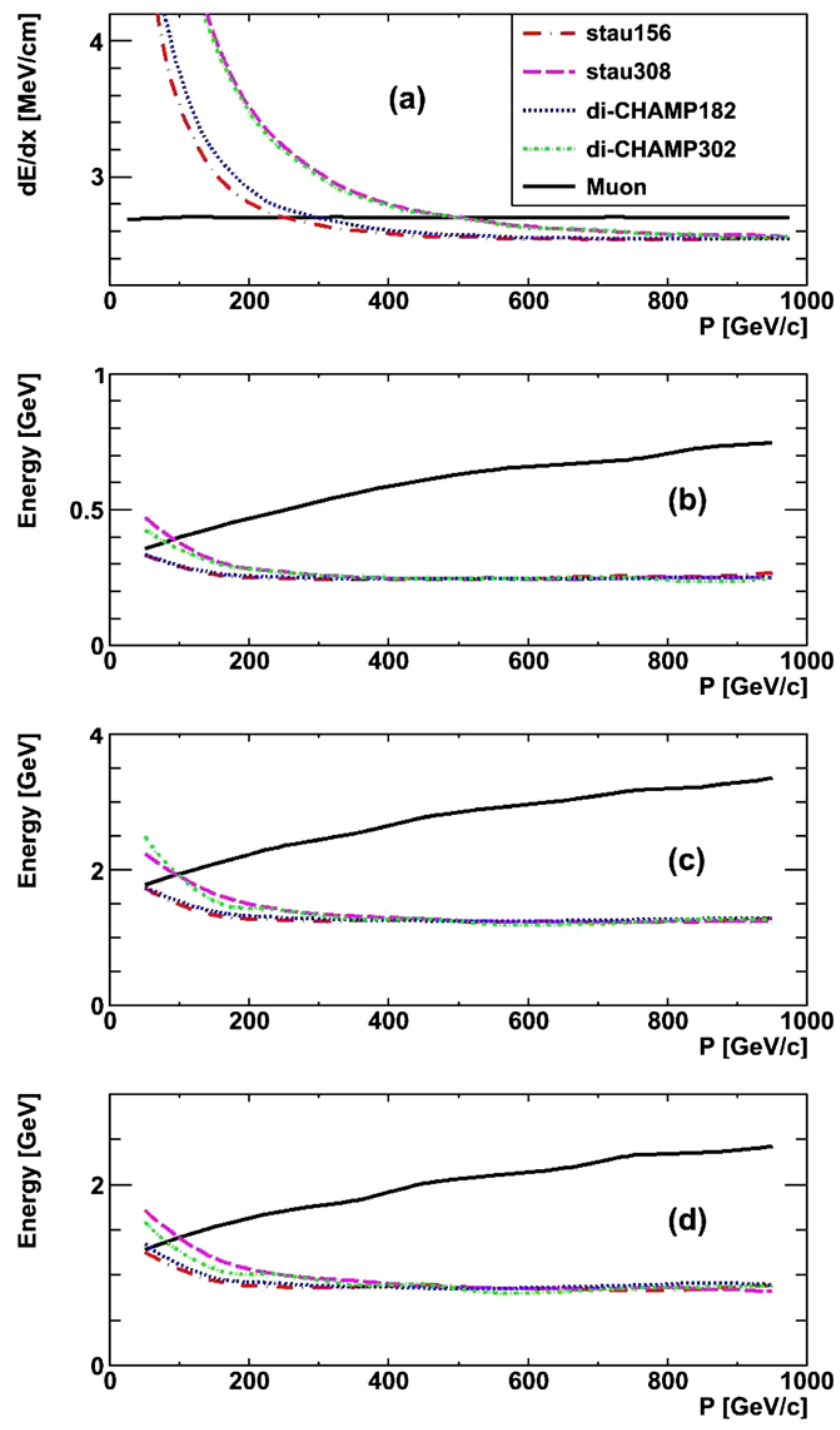

Fig. 7 The average (a) Tracker $d E / d x$, (b) energy deposition in Ecal, (c) energy deposition in Hcal, and (d) difference between Hcal and Ecal in energy deposition, as a function of CHAMP/muon momentum for four different CHAMP model/mass points and background muons. Further analysis in this paper will concentrate on the high momentum $(\gtrsim 500 \mathrm{GeV} / \mathrm{c}$ ) region

Figure 8 shows the BDT discriminant distribution for the stau156 model and backgrounds after our simple selection. We see a very clear separation between high speed CHAMPs and background muons. The di-CHAMP182 model has a behavior very similar to the one of the stau 156 model. We estimate the significance of the signal using

$S_{\mathrm{cL}}=\sqrt{2\left((S+B) \ln \left(1+\frac{S}{B}\right)-S\right)}$,

where $S$ and $B$ are the number of signal and background events [39] that remain after a cut on the BDT discriminant. The cut is chosen by maximizing $S_{\mathrm{cL}}$. 


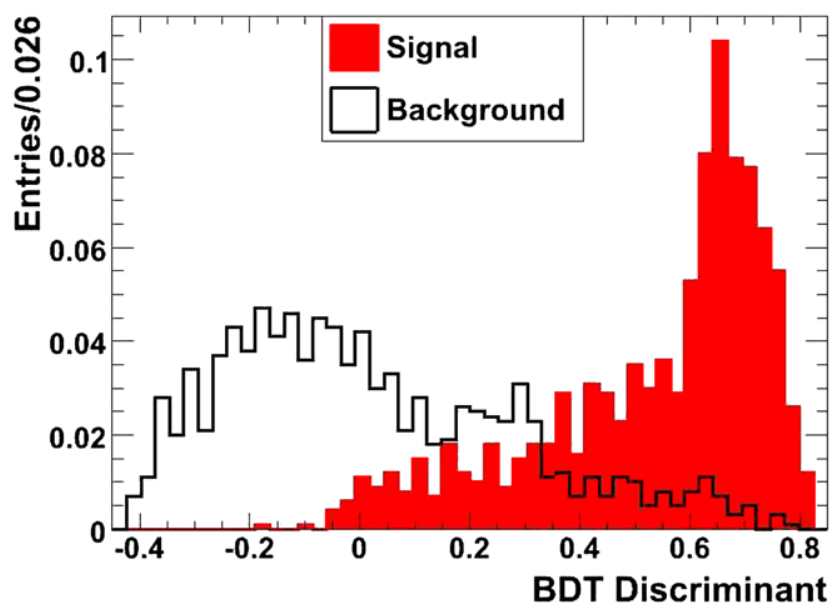

Fig. 8 Distributions of the BDT discriminant for stau156 signal (solid) and background (empty)

We use $S_{\mathrm{cL}}$ to illustrate the expected improvement in sensitivity of the BDT method relative to the conventional one. A more precise estimate of the sensitivity of the LHC experiments for observing a possible CHAMP signal would require a full detector simulation. However, the main point we wish to make is that a better use of the energy depositions can enhance significantly the discovery potential of the LHC using the early data. The quantity $S_{\mathrm{cL}}$ is sufficient to make this point.

\section{Application of the BDT discriminant}

\subsection{Discovery potential for CHAMPs}

For the two benchmark models, Table 4 compares the $S_{\mathrm{cL}}$ values expected using a simple counting method with those expected from the BDT method. We have assumed an integrated luminosity of $200 \mathrm{pb}^{-1}$, collected at an LHC center of mass energy of $10 \mathrm{TeV}$. We note that a simple counting experiment can already give an $S_{\mathrm{cL}}$ as high as $5.6 \sigma$ (stau156 model with nominal background) using our selection of high momentum tracks within the muon acceptance. Even without looking at the energy deposition differences between muons and CHAMPs, a low mass CHAMP discovery is already possible for some model/mass points.

Table 4 Signal significance assuming $200 \mathrm{pb}^{-1}$ data at $\sqrt{s}=10 \mathrm{TeV}$ LHC, using simple counting method or BDT

\begin{tabular}{llllll}
\hline Model & $\begin{array}{l}\text { Nominal } \\
\text { background }\end{array}$ & & \multicolumn{2}{l}{$\begin{array}{l}\text { Double nominal } \\
\text { background }\end{array}$} \\
\cline { 2 - 3 } \cline { 5 - 6 } & Counting & BDT & & Counting & BDT \\
\hline stau156 & $5.6 \sigma$ & $8.3 \sigma$ & $4.4 \sigma$ & $7.1 \sigma$ \\
di-CHAMP182 & $4.2 \sigma$ & $6.7 \sigma$ & $3.2 \sigma$ & $5.8 \sigma$ \\
\hline
\end{tabular}

The background could be larger than our estimate due to several effects. The PDFs are not well constrained at LHC energies. Our simplified GEANT4 simulation does not include track momentum mis-measurement that could cause moderate momentum (100-500 GeV) muons to reconstruct at larger momentum $(>500 \mathrm{GeV})$. A full detector simulation and reconstruction as well as comparison to real data is necessary to incorporate these effects. However, Table 4 also shows the effect of doubling the background estimate to simulate these types of effects. The larger background would lower the significance of both methods. However, the BDT discriminant can ensure discoveries ( $>5 \sigma$ significance) for the stau156 and di-CHAMP182 benchmarks, even if the background were larger than our nominal estimate by a factor of two.

The method proposed here has very high detection efficiency because the CHAMP is moving very fast and therefore, like a high $p_{T}$ muon, its trigger and reconstruction efficiencies are very high at CMS and ATLAS. (For $\beta$ less than $\sim 0.6$, however, the trigger and reconstruction efficiencies for CHAMPs drop significantly at CMS [18] and ATLAS [19].) Moreover, the new method can use high momentum cosmic muons to evaluate instrumental effects related to energy depositions. Recent results from the CMS collaboration show the GEANT4 simulation is consistent with detector response to cosmic muons [40-42].

We then compare the expected significance of the conventional low $\beta$ method, the high $p$ counting method, and the new BDT method for the two benchmark models. Table 5 lists the $S_{\mathrm{cL}}$ values from the three methods, assuming $200 \mathrm{pb}^{-1}$ data from $10 \mathrm{TeV}$ running at the LHC, for several mass points. The efficiency is obtained from the number of CHAMP tracks in the slow $(0.6<\beta<0.8$ and $|\eta|<0.8$ and $p_{T}>40 \mathrm{GeV} / \mathrm{c}$ ) or fast CHAMP regions (as defined in this paper), divided by the total number of events. We assume

Table 5 Signal significance from the conventional, counting, and BDT method, assuming $200 \mathrm{pb}^{-1}$ data from $10 \mathrm{TeV}$ LHC. The efficiency for the events to fall within the basic selection (see text) is given in parenthesis

\begin{tabular}{llll}
\hline $\begin{array}{l}\text { CHAMP mass } \\
\left(\mathrm{GeV} / \mathrm{c}^{2}\right)\end{array}$ & Conventional & Counting & New BDT \\
\hline $\begin{array}{l}\text { mGMSB stau } \\
156\end{array}$ & $>6.8 \sigma(0.20)$ & $5.6 \sigma(0.32)$ & $8.3 \sigma$ \\
200 & $>3.0 \sigma(0.24)$ & $2.2 \sigma(0.40)$ & $3.9 \sigma$ \\
247 & $>1.3 \sigma(0.28)$ & $0.8 \sigma(0.42)$ & $1.4 \sigma$ \\
308 & $>0.5 \sigma(0.33)$ & $0.3 \sigma(0.49)$ & $0.5 \sigma$ \\
di-CHAMP resonance & & \\
182 & $>4.2 \sigma(0.19)$ & $4.2 \sigma(0.41)$ & $6.7 \sigma$ \\
242 & $>2.0 \sigma(0.25)$ & $1.9 \sigma(0.57)$ & $3.3 \sigma$ \\
302 & $>1.0 \sigma(0.28)$ & $1.0 \sigma(0.72)$ & $1.4 \sigma$ \\
\hline
\end{tabular}




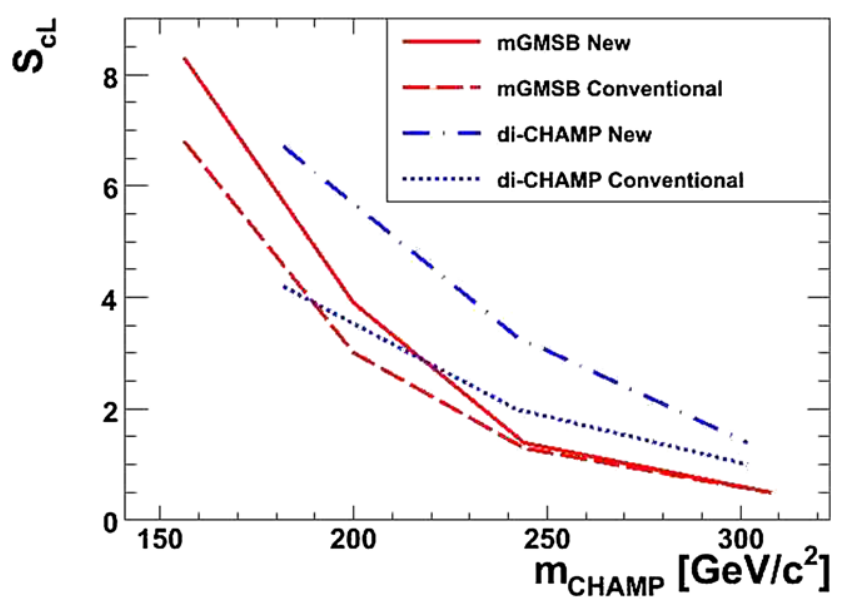

Fig. 9 (Color online) Significance of the new and conventional search methods as a function of the CHAMP mass for the two models explored: mGMSB SPS7 (red) and di-CHAMP (blue)

$100 \%$ trigger and reconstruction efficiency for CHAMPs in both the conventional and BDT methods, a background of $<1$ event for the conventional method [18], and our nominal background estimate for the new method.

Figure 9 shows the significance of the new and conventional methods as a function of CHAMP mass. We see that for the masses of interest for early LHC running, the high momentum analysis has comparable or better discovery potential. The curve for the conventional method is a rough estimate and it could turn out to be larger, but we do not expect it to be radically different. The two search methods are complementary as they explore different regions of signal acceptance. Therefore, the discovery potential could be enhanced further by combining the two methods, taking due account of correlations.

Although this paper uses CMS as a model detector, the method is applicable to ATLAS, which has similar Hcal and Ecal energy resolutions as CMS [43]. Although ATLAS does not measure the Tracker $\mathrm{dE} / \mathrm{dx}$ in the same way as CMS, the number of hits in the Transition Radiation Tracker (TRT) will give similar discriminating power. This is because a high speed muon results in more high threshold hits, but a high speed CHAMP (still in the $\beta \gamma=1-10$ region) only gives about 2 hits, as pointed out in [44]. In addition, the technique could be extended to include information from other ATLAS or CMS detectors such as proposed in [45].

\subsection{Distinguishing di- $\mu$ signal from di-CHAMP or CHAMP- $\mu$ signal}

In the di-CHAMP model, CHAMPs can originate from the decay of a heavy resonance. Therefore, it is interesting to look at the invariant mass spectrum (calculated assuming the muon mass) for those events. As shown in Fig. 10, after the initial selection used in this analysis (not including the

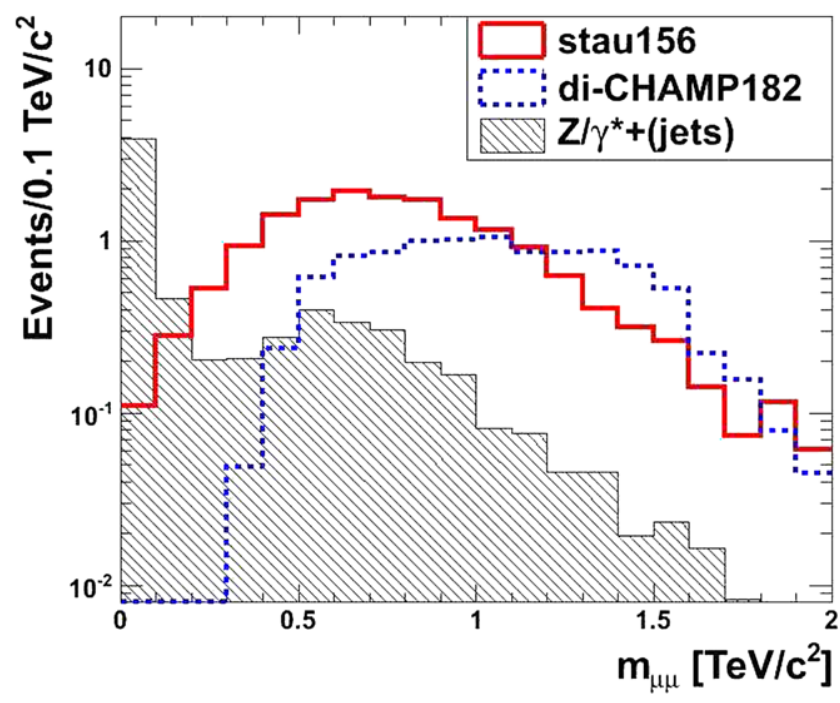

Fig. 10 Di- $\mu$ mass for main background and signals (stau156 and di-CHAMP182 models, unstacked) after initial selections in this analysis. The di- $\mu$ Mass is smeared with mass resolution in [46]. Plot is scaled to $200 \mathrm{pb}^{-1} 10 \mathrm{TeV}$ LHC data

BDT), we see a broad excess above $500 \mathrm{GeV} / \mathrm{c}^{2}$ in the $\mathrm{di}-\mu$ mass spectrum. Previous Monte Carlo studies have shown some evidence that a peak may be observable [47], but it is not seen here with this benchmark model.

Even for CHAMPs that do not originate from a common resonance, both models predict an excess of events at high di- $\mu$ mass (Fig. 10). Therefore, an analysis searching the di- $\mu$ spectrum above the $Z$ peak (for evidence of a $Z^{\prime}$, compositeness, Randall-Sundrum gravitons, etc.) will need to determine whether any observed excess arises from muons or from CHAMPs. Our new method can easily distinguish events with two CHAMPs from those with two muons. The method will also be useful in identifying CHAMP-muon resonances such as those suggested in [7].

\section{Conclusion}

We propose a way to search for high speed CHAMPs at the LHC experiments to complement the conventional slowly moving CHAMP searches. The SM background can be kept to a very low level using simple selection criteria. With $200 \mathrm{pb}^{-1}$ data collected at $10 \mathrm{TeV}$ during the first run of the LHC, a simple counting experiment already has the possibility to discover a stau with mass just above $140 \mathrm{GeV} / \mathrm{c}^{2}$. To improve the signal-background separation, we construct a boosted decision tree whose inputs are the energy depositions from muon-like tracks. This discriminant can improve significantly the discovery potential during early LHC running, which is our principal goal. By combining the two search methods, one can, in principle, increase the discovery potential further. Finally, the BDT discriminant pro- 
vides an experimental tool to distinguish CHAMP models (di-CHAMP, CHAMP-muon resonance models or nonresonance CHAMP models) from models with di-muon excesses in standard high mass di- $\mu$ searches.

Acknowledgements This work was supported by the US Department of Energy under grant No. DE-FG02-95ER40896. We thank Can Kilic, Takemichi Okui and Raman Sundrum for providing their model code and Thomas Phillips for discussion of the interpretation of the CDF CHAMP search. We also thank Harrison Prosper for very helpful discussions on multivariate analysis techniques.

\section{References}

1. G. Giudice, R. Rattazzi, Phys. Rep. 322, 419 (1999)

2. G. Giudice, A. Romanino, Nucl. Phys. B 699, 65 (2004)

3. M. Byrne, Phys. Lett. B 583, 309 (2004)

4. M. Carena et al., Phys. Rev. D 66, 115010 (2002)

5. J. Feng, A. Rajaraman, B. Smith, Phys. Rev. D 74, 015013 (2006)

6. C. Kilic, T. Okui, R. Sundrum, J. High Energy Phys. 02, 018 (2010)

7. A.D. Simone, J. Fan, V. Sanz, W. Skiba, Phys. Rev. D 80, 035010 (2009)

8. S. Kaneko, J. Sato, T. Shimomura, O. Vives, M. Yamanaka, Phys. Rev. D 78, 116013 (2008)

9. C.F. Berger et al., J. High Energy Phys. 0902, 023 (2009)

10. P. Achard et al. (L3 Collaboration), Phys. Lett. B 517, 75 (2001)

11. G. Abbiendi et al. (OPAL Collaboration), Phys. Lett. B 572, 8 (2003)

12. A. Heister et al. (ALEPH Collaboration), Eur. Phys. J. C 25, 339 (2002)

13. J. Abdallah et al. (DELPHI Collaboration), Eur. Phys. J. C 31, 421 (2003)

14. LEP SUSY Working Group, ALEPH, DLEPHI, L3 and, OPAL collaborations, note LEPSUSYWG/02-05.1, http://lepsusy.web. cern.ch/lepsusy/Welcome.html

15. T. Aaltonen et al. (CDF Collaboration), Phys. Rev. Lett. 103, $021802(2009)$

16. V.M. Abazov et al. (D0 Collaboration), Phys. Rev. Lett. 102, 161802 (2009)

17. M. Fairbairn, A.C. Kraan, D.A. Milstead, T. Sjostrand, P. Skands, T. Sloan, Phys. Rep. 438, 1 (2007)
18. CMS Collaboration, CMS-PAS-EXO-08-003, available at http:// cdsweb.cern.ch/record/1152570? $1 \mathrm{n}=\mathrm{en}$

19. S. Tarem et al., Eur. Phys. J. C 62, 281 (2009)

20. B.C. Allanach et al., Eur. Phys. J. C 25, 113 (2002)

21. F. Paige et al., arxiv:hep-ph/0312045

22. T. Sjostrand, S. Mrenna, P. Skands, J. High Energy Phys. 0605, 026 (2006)

23. E. Boos et al. (CompHEP Collaboration), Nucl. Instrum. Methods A 534, 250 (2004)

24. T. Han et al., Phys. Rev. D 67, 095004 (2003)

25. L. Randall, R. Sundrum, Phys. Rev. Lett. 83, 4690 (1999)

26. D. Choudhury, S. Majhi, V. Ravindran, Nucl. Phys. B 660, 343 (2003)

27. D. Feldman, Z. Liu, P. Nath, J. High Energy Phys. 0611, 007 (2006)

28. E.J. Eichten, K.D. Lane, M.E. Peskin, Phys. Rev. Lett. 50, 811 (1983)

29. F. Abe et al. (CDF Collaboration), Phys. Rev. Lett. 79, 2198 (1997)

30. Private Communication with Thomas Phillips

31. J. Huston et al., Phys. Rev. Lett. 77, 444 (1996)

32. C. Amsler et al. (Particle Data Group), Phys. Lett. B 667, 1 (2008)

33. R. Adolphi et al. (CMS Collaboration), JINST 3, S08004 (2008)

34. J. Allison et al., IEEE Trans. Nucl. Sci. 53, 270 (2006)

35. W. Adam et al. (CMS Tracker Collaboration), JINST 4, P05004 (2009)

36. G.L. Bayatian et al. (CMS Collaboration), CMS Physics Technical Design Report, CERN/LHCC/2006-001

37. V.M. Abazov et al. (D0 Collaboration), Phys. Rev. Lett. 102, 192002 (2009)

38. A. Hocker et al., PoS ACAT 040 (2007)

39. G.L. Bayatian et al. (CMS Collaboration), J. Phys. G, Nucl. Part. Phys. 34, 995 (2007)

40. CMS Collaboration, arXiv:0911.4996 [physics.ins-det]

41. CMS Collaboration, arXiv:0911.5397 [physics.ins-det]

42. CMS Collaboration, arXiv:0911.4991 [physics.ins-det]

43. G. Aad et al. (ATLAS Collaboration), arXiv:0901.0512 [hep-ex]

44. A.C. Kraan, J.B. Hansen, P. Nevski, Eur. Phys. J. C 49, 623 (2007)

45. C. Albajar et al. (RD5 Collaboration), Nucl. Instrum. Methods A 364, 473 (1995)

46. CMS Collaboration, CMS-PAS-SBM-07-002, available at http:// cdsweb.cern.ch/record/1152564? ln=en

47. C. Kilic, T. Okui, arXiv:1001.4526 [hep-ph] 